Le precedenti osservazioni hanno presentato qualche difficoltà a cagione della forma allungata del nucleo della Cometa. Come punto di mira si è procurato ogni volta di prendere il centro della massa nebulosa fusiforme, benchè non fosse certa la coincidenza di questo punto col centro di gravità.

Le riduzioni sono state eseguite colla cooperazione del Dr. Porro. Furono fatte anche annotazioni sull' aspetto della Cometa, e fu delineato il contorno della coda e delle sue varie appendici: ma la pubblicazione di questi risultati richiede un certo numero di disegni e perciò si riserva ad un' altra occasione.

Osservatorio Reale di Brera in Milano $r 884$ Settembre 24.

G. V. Schiaparelli.

\title{
Observations of Comet 1884 II (Barnard)
}

made at the Royal Observatory, Cape of Good Hope.

(Communicated by David Gill L. L. D. F. R. S. Her Majesty's Astronomer).

The following observations were made, with the 7 inch Equatoreal and Repsold-Filar-Micrometer, by $\mathrm{Mr}$. W. H. Finlay, Chief Assistant. The comet was so situated that meridian observations of the comparison stars could always be secured with the transit circle within a few nights after their comparison with the Comet.

The observations are corrected for the effects of refraction.

The Comet throughout presented the appearance of a small diffused nebulous mass. A nucleus was seen by glimpses on July 28 , but the bright moonlight on following nights prevented more than a suspicion of its existence. About the end of July the point of greatest condensation (within which the nucleus had been seen on July 28 ) was slightly towards the following part of the mass; this was the point observed. In August the appearance of the Comet was reduced by moonlight to a small round mass slightly brighter towards the centre _.. the centre of this mass was observed. The Comet presented a similar appearance in September and was similarly observed.

An apparent increase of brightness on September I $_{5}$ and following days, compared with the appearance of the Comet in the end of August, was very striking. 'The Comet as a rule was faint and difficult to observe in August, probably from its then being projected on the milky way as a back ground.

Observations of Comet, I884 I (Pons I812) have been secured on 43 nights from January 16 to April 29 by Mr. Finlay, and will be published so soon as the meridian observations of the comparison stars have been completed.

\begin{tabular}{|c|c|c|c|c|c|c|c|c|c|c|c|c|c|c|c|}
\hline \multicolumn{2}{|l|}{1884} & \multicolumn{3}{|c|}{ Cape M. T. } & $\triangle \mathrm{RA}$ & $\triangle \mathrm{NPD}$ & Comp. & \multicolumn{3}{|c|}{ RA. app. } & $\log$ f.p. & \multicolumn{2}{|c|}{ NPD. app. } & \multirow{2}{*}{$\begin{array}{c}\log \text { f.p. } \\
8.580\end{array}$} & \multirow[t]{2}{*}{ Star } \\
\hline July 2 & 24 & $10^{h}$ & $8^{m}$ & 8.1 & $-0^{\mathrm{mn}} 13^{\mathrm{s}} .00$ & $+6^{\prime} 57^{\prime \prime} \mathbf{I}$ & 4.4 & I $6^{\mathrm{h}}$ & $5^{1}$ & 30.56 & 8.617 & I $27^{\circ}$ I 4 & $49 . .1$ & & \\
\hline & 26 & IO & $3^{8}$ & 4.0 & $+430.3^{8}$ & -629.0 & 4.4 & I 6 & 10 & I 4.75 & 8.668 & $127 \quad 15$ & $34 . r$ & 8.839 & $b$ \\
\hline & 27 & 10 & 54 & 54.1 & to 10.09 & $\begin{array}{ll}-1 & 1 \\
4.3\end{array}$ & 16.16 & I 6 & I 2 & 41.63 & 8.703 & $127 \times 5$ & $35 \cdot 5$ & 8.987 & $c$ \\
\hline & 28 & I I & 6 & I 5.8 & -0 I $4.1 \mathrm{I}$ & +633.3 & I 6.16 & I 6 & I 5 & 14.61 & 8.725 & I 27 I 5 & 30.4 & 9.072 & Stone 8897 \\
\hline & 29 & I I & Io & $3^{2.2}$ & +10.99 & -226.8 & 1 6.16 & 16 & I 7 & 52.45 & 8.734 & I 27 I 5 & I $5 . \mathrm{I}$ & 9.107 & Stone 8913 \\
\hline & 30 & I I & 5 & I 5.7 & to $474 \mathrm{I}$ & +159.5 & I 6.16 & r 6 & 20 & 33.87 & 8.727 & $127 \quad 14$ & $4^{6.8}$ & 9.079 & $d$ \\
\hline Aug. & 1 & 10 & I 9 & $53 \cdot 3$ & -o 7.04 & +131.0 & 10.6 & I 6 & 26 & 5.69 & 8.644 & 127 I 3 & I 9.6 & $8.59 \mathrm{I}$ & $\varepsilon$ \\
\hline & 5 & 9 & 35 & $49 \cdot 5$ & to 32.05 & to 53.5 & I 6.16 & 16 & $3^{8}$ & $8.3^{2}$ & $8.53^{\circ}$ & 1277 & 6.2 & 7.699 & $f$ \\
\hline & 6 & 9 & 53 & $49 \cdot 7$ & to 30.12 & +218.8 & I6.1 6 & 16 & $4 I$ & 24.38 & 8.580 & 127 & 44.4 & 8.447 & $g$ \\
\hline & 7 & IO & Io & 18.4 & to 21.60 & +131.6 & I 6.16 & 16 & 44 & 43.78 & 8.633 & I 27 & 3.0 & 8.708 & $h$ \\
\hline & 8 & 10 & Io & 5.0 & $-0 \quad 20.07$ & -046.7 & 20.16 & I 6 & 48 & 4.67 & 8.633 & I $26 \quad 59$ & 9.8 & 8.708 & $i$ \\
\hline & I I & Io & 4 & 50.4 & -022.80 & -12.6 & 20.16 & I 6 & $5^{8}$ & 29.35 & 8.622 & I 2648 & 5.2 & 8.690 & $k$ \\
\hline & I 3 & 10 & I 4 & 35.2 & to 23.13 & $-3 \quad 30.7$ & 20.16 & I 7 & 5 & 45.67 & 8.646 & I 2638 & 44.6 & 8.820 & $l$ \\
\hline & 14 & 9 & 45 & $9 \cdot 5$ & -0 21.98 & $-3 \quad 17.2$ & I 2.12 & I 7 & 9 & 23.79 & 8.580 & I 2633 & 25.7 & $8.53^{2}$ & $m$ \\
\hline & 17 & 9 & 47 & $3^{6.8}$ & to 29.46 & $+0 \quad 0.8$ & I 6.16 & I 7 & 20 & $47 \cdot 5^{8}$ & 8.576 & $126 \quad 14$ & 54.6 & 8.580 & $n$ \\
\hline & I 9 & I0 & I0 & 4.8 & $-0 \quad 17.79$ & -237.2 & 15.16 & I 7 & 28 & 40.96 & 8.635 & 126 & 4.9 & 8.833 & $o$ \\
\hline & 20 & 10 & I 2 & 46.7 & -025.26 & - $0 \quad$ I 2.9 & I 6.8 & I 7 & $3^{2}$ & 39.96 & 8.640 & I $25 \quad 5^{2}$ & 10.9 & 8.771 & $p$ \\
\hline & 22 & 10 & 26 & 1.8 & to 10.08 & $+45^{2.3}$ & I 6.16 & I 7 & $4^{\circ}$ & 44.45 & $8.66_{7}$ & 12534 & 24.9 & 9.000 & $q$ \\
\hline & 23 & Io & 4 & 39.6 & to 35.86 & +54.2 & 16.16 & I 7 & 44 & 44.83 & 8.617 & 12524 & $54 . \mathrm{I}$ & $8.85 \mathrm{I}$ & Y. 7433 \\
\hline Sept. I & 15 & IO & I 9 & 31.8 & to 11.50 & +57.9 & 24.18 & I 9 & 20 & 3.95 & 8.614 & 11937 & $5 \cdot 7$ & $9.23^{6}$ & $\mathrm{AOe}_{2}$ I 9534 \\
\hline & 17 & IO & 27 & I 3.5 & $-0 \quad 51.09$ & +2 1 2.9 & 16.16 & 19 & 28 & $0 . ; \mathbf{I}$ & 8.626 & I 1857 & I 7.5 & 9.272 & $\mathrm{AOE}_{2} 19737$ \\
\hline & 21 & 9 & 44 & 44.1 & -016.20 & -226.6 & 17.12 & 19 & 43 & 25.02 & 8.5 I 3 & 11735 & $4^{8.0}$ & 9.225 & $\mathrm{AOe}_{2} 19997$ \\
\hline & 22 & IO & I 2 & I 2.9 & to 54.93 & to $7 \cdot 3$ & I 2.1 6 & 19 & 47 & $16.3^{6}$ & 8.587 & I I 7 I 4 & 19.4 & 9.295 & $\mathrm{AOe}_{2} 20039$ \\
\hline
\end{tabular}


The comet was also observed with the transit-circle by Mr. Finlay on August 14 and 16 , with the following result:

\begin{tabular}{|c|c|c|c|c|}
\hline I 884 & Cape M.'T. & RA. app. & NPD. app. & $\log$ f. p. \\
\hline $\begin{aligned} \text { Aug. } & 14 \\
& 16\end{aligned}$ & $\begin{array}{l}7^{\mathrm{h}} 34^{\mathrm{m}} 48.8 \\
734 \quad 26.0\end{array}$ & $\begin{array}{lll}17^{\mathrm{h}} & 9^{\mathrm{m}} & 6^{\mathrm{s}} \mathrm{1} 9 \\
\mathrm{1} 7 & 16 & 36.39\end{array}$ & 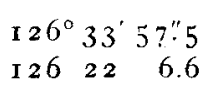 & $\begin{array}{l}8.68 \mathrm{I}_{\mathrm{n}} \\
8.644 \mathrm{n}\end{array}$ \\
\hline
\end{tabular}

\section{$\mathrm{N} o \mathrm{t}$ e s.}

July 24. Comet exceedingly faint and difficult - hazy.

28. Comet much easier to-night; distinct appearance of nucleus or condensation.

Aug. I. Comet very faint; bright moonlight.

5. Moon very bright, but fair observations.

7. Bright moonlight; air very damp and hazy; Comet very faint.
Aug. I 3. Comet faint.

I 9. Comet very faint; very difficult.

20. Comet exceedingly faint, scarcely possible to observe at all.

22. Fair observations.

Sept. 15. Comet much easier and brighter than when last observed.

Adopted Right Ascensions and North Polar Distances of Stars observed with the Comet.

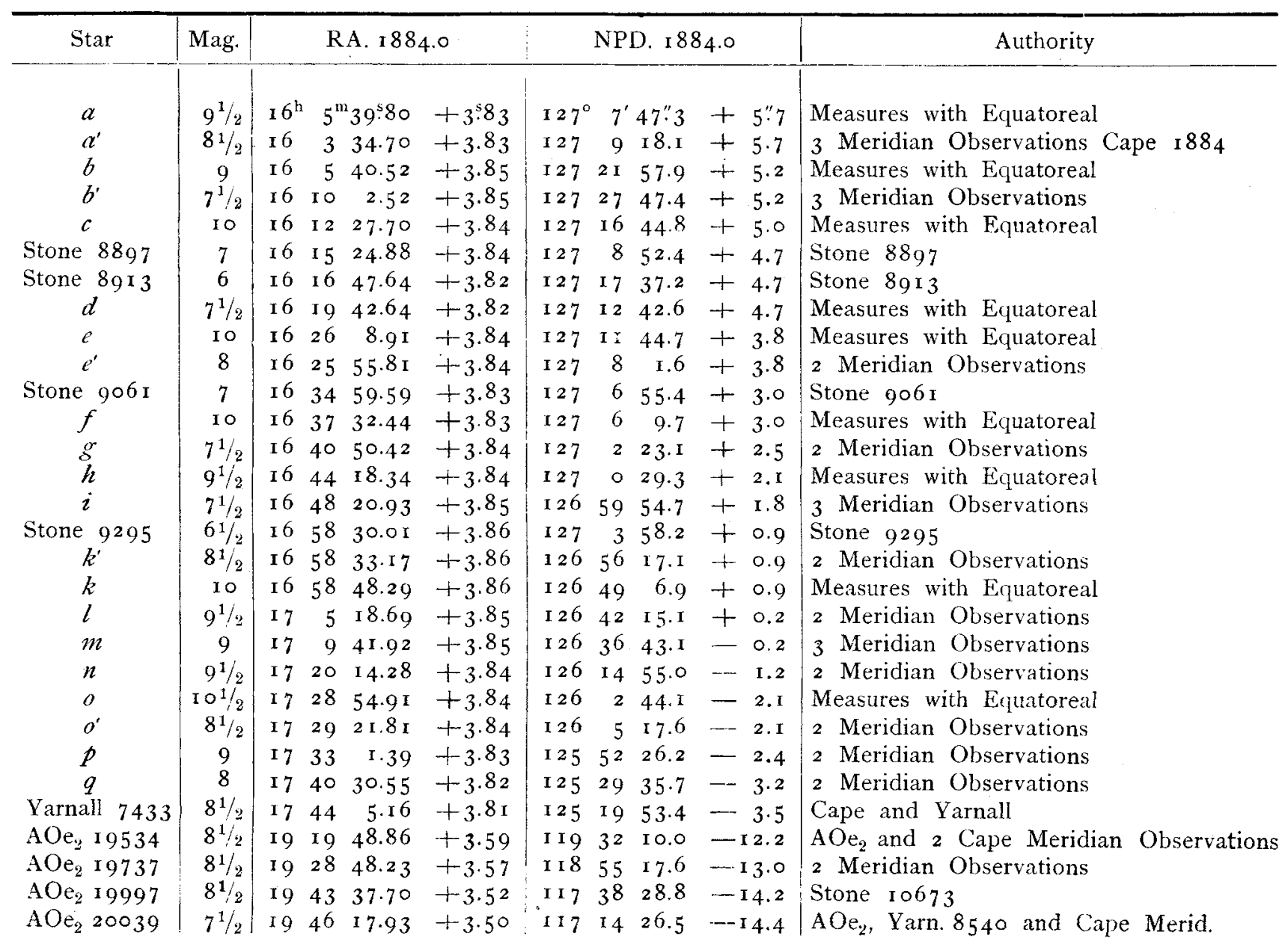

The following Table gives the differences of RA. and NPD. between the Comparison stars and brighter stars, observed with the Equatoreal. 


\begin{tabular}{|c|c|c|c|c|}
\hline Star & Mag. & $\Delta \mathrm{RA}$. & $\triangle \mathrm{NPD}$. & Comp. with $*$ \\
\hline$a$ & $9^{1 / 2}$ & $+2^{\mathrm{m}} 5^{\mathrm{s}} \mathrm{10}$ & -. I' $30^{\prime \prime} 8$ & $a^{\prime}$ \\
\hline$b$ & 9 & -422.00 & $\begin{array}{ll}-5 & 49.5\end{array}$ & $b^{\prime}$ \\
\hline$c$ & Io & $-2 \quad 57.55$ & +751.9 & Stone 8897 \\
\hline$c$ & 10 & $--4 \quad 19.75$ & $-0 \quad 52.0$ & Stone 8913 \\
\hline$d$ & $7^{1 / 2}$ & +255.00 & $--4 \quad 54.6$ & Stone 8913 \\
\hline$e$ & 10 & +o 13.10 & +343.1 & $e^{\prime}$ \\
\hline
\end{tabular}

\begin{tabular}{|c|c|c|c|c|}
\hline Star & Mag. & $\Delta \mathrm{RA}$. & $\triangle \mathrm{NPD}$. & Comp. with * \\
\hline$f$ & 10 & $+2^{\mathrm{m}} 32^{5.8} 5$ & $-0^{\prime} 45^{\prime \prime} \cdot 7$ & Stone $906 \mathrm{I}$ \\
\hline$h$ & $9^{1 / 2}$ & +328.30 & - I 55.0 & $g$ \\
\hline$h$ & $9^{1 / 2}$ & $\begin{array}{ll}-4 \quad 2.95\end{array}$ & to 35.5 & $i$ \\
\hline$k$ & 10 & to 15.12 & $-7 \times 0.2$ & $k^{\prime}$ \\
\hline$k^{\prime}$ & $81 / 2$ & to 3.10 & $-7 \quad 40.7$ & Stone 9295 \\
\hline$o$ & I $0^{1 / 2}$ & $-0 \quad 26.90$ & 233.5 & $o^{\prime}$ \\
\hline
\end{tabular}

David Gill.

Beobachtungen des Cometen 1884 II (Barnard).

\begin{tabular}{|c|c|c|c|c|c|c|c|c|}
\hline I 884 & M.Z.Strassb. & $\Lambda \alpha$ & $\Delta \delta$ & $\alpha$ app. & $\log p \cdot A$ & $\delta$ app. & $\log p .4$ & $*$ \\
\hline Oct. I9 & $7^{\mathrm{h}} 31^{\mathrm{m}} 42^{\mathrm{s}}$ & - $0^{\mathrm{m}} 8.43$ & $+3^{\prime} 48{ }^{\prime \prime} 4$ & $21^{\mathrm{h}} 16^{\mathrm{m}} 53^{\mathrm{s}} \mathrm{I}^{8}$ & 8.209 & $-\mathrm{I} 7^{\circ} 3 \mathrm{I}^{\prime} \quad 3^{\prime \prime} 2$ & 0.909 & $\mathbf{I}$ \\
\hline 2 I & $7595^{2}$ & - I $\quad 13.75$ & -0 29.5 & $\begin{array}{lll}21 & 22 & 41.74\end{array}$ & 8.843 & - I $6484^{8} 8.5$ & 0.905 & 2 \\
\hline 23 & $741 \quad 2$ & +2 I 4.43 & +5 I $9 . \mathrm{I}$ & $\begin{array}{lll}21 & 28 & 18.58\end{array}$ & 8.594 & 737.9 & 0.904 & 3 \\
\hline
\end{tabular}

Mittlere Oerter der Vergleichsternefür 1884.0.

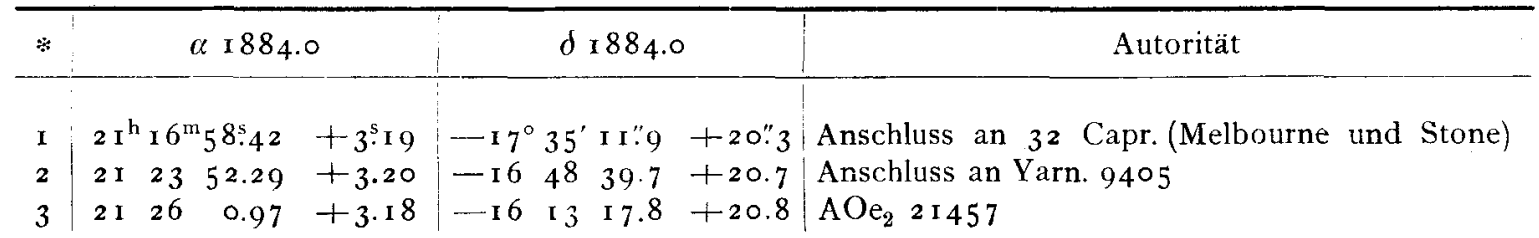

Die Beobachtungen verursachten in den letzten Tagen schon bedeutende Schwierigkeiten, so dass ich nicht sicher bin, ob ich nach Verschwinden des Mondes zu weiteren Beobachtungen im Stande sein werde.

Strassburg 1884 Oct. 3 I.

Wilhelm Schur.

\section{Approximate elliptic elements of Comet 1884 II (Barnard).}

I computed a parabolic orbit for this comet in August but was unable to represent the middle place satisfactorily. As a similar result was found in Europe and as Mr. Stechert's ephemeris in Astr. Nachr. Nr. 2609 shewed large discordances from my later observations, I have computed elliptic elements with the following result. The observations used, viz. July 27, August 22 and September 17, were corrected for parallax from the parabolic orbit. The approximations to $Q$ were not carried as far as perhaps they should have been, but the approximation was quite close enough to shew whether the comet was moving in an ellipse and, if so, to give a very fair value of the periodic time.

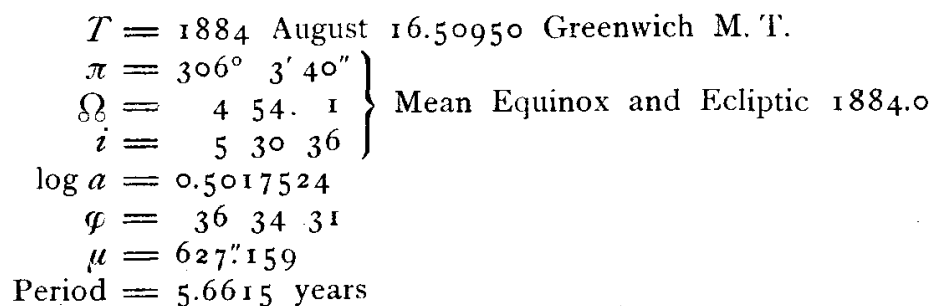

These elements leave a discordance $(\mathrm{C}-\mathrm{O})$ in the middle place of -8 " in longitude and - $\mathbf{I}^{\prime \prime}$ in latitude. The month of October, since the Moon drew away from the evening sky, has been completely clouded until last night Oct. I4, when I secured a fair comparison of the comet with AOe 2 II 5 I. This observation compared with the above elements gives the discordance $(\mathrm{C}-\mathrm{O})$ :

$$
\mathrm{d} \alpha \cos \delta=-5^{\mathrm{s}} \cdot \mathrm{d} \delta=-\mathrm{I} \mathrm{x}^{\prime \prime}
$$

Royal Observatory, Cape of Good Hope $188_{4}$ Oct. 15.

W. H. Finlay. 